\title{
THE SMALL PULMONARY ARTERIES STUDIED BY A NEW INJECTION METHOD
}

\author{
BY \\ W. R. L. JAMES, G. M. OWEN, AND A. J. THOMAS \\ From the Institute of Pathology, Welsh National School of Medicine, Cardiff \\ Radiotherapy Centre, and Llandough Hospital (United Cardiff Hospitals)
}

Received February 10, 1960

The object of this work was to try and advance our knowledge of the changes in the pulmonary vascular bed that lead to right ventricular hypertrophy in certain cases of cardiac and respiratory disease. We had noted in previous work some unexplained examples of right ventricular hypertrophy. Some cases of pulmonary heart disease had greatly hypertrophied right ventricles with apparently little gross change in the lungs. Other cases in congestive heart failure had gross changes in the lungs and apparently small right ventricles, but in some of these we were able to demonstrate relative right ventricular hypertrophy by weight when the ventricles were dissected, the left ventricle being very small, and the right ventricle the heavier of the two (Thomas and James, 1958). Histological changes in the pulmonary vascular bed have been described by many workers (Brenner, 1935; Parker, 1940; McKeown, 1952). Injection studies in conditions such as mitral stenosis and congenital heart disease have suggested that the pulmonary arterial bed is reduced (Short, 1957; Evans and Short, 1958). Physiological studies have shown that acute anoxia and some pharmacological agents can produce a transient restriction in the pulmonary vascular bed (Motley et al., 1947; Borst et al., 1957).

Where does this "shut-down" take place, what is its character, and how much permanent effect does it produce? Lung injection studies in pulmonary angiography of mitral stenosis (Goodwin et al., 1952; Davies et al., 1953) and in post-mortem specimens (Short 1957), have shown the bare or pruned tree picture indicating that the smaller vessels of the pulmonary arterial bed have been narrowed or obliterated. The assessment of these pictures was visual and we have had no actual measure of the change: consequently in some cases of lesser degree it has been difficult to decide how much change has taken place. We considered this problem and have developed a method of injecting lung specimens in such a way as to obtain a measure of the volume of the arterial bed in the peripheral parts of these lungs (Thomas et al., 1959). We chose to study the peripheral portions because it is rare to find arteries exceeding $2 \mathrm{~mm}$. in diameter in these parts and we were interested in the patency of the arteries of less than $2 \mathrm{~mm}$. diameter.

\section{METHOD}

The injection mixture used is made up by adding propyliodane aqueous suspension (dionosil aqueous) $60 \mathrm{ml}$. to a solution of $10 \mathrm{~g}$. of agar in $440 \mathrm{ml}$. water at a temperature of $60^{\circ} \mathrm{C}$. Sodium acetate $0.5 \mathrm{~g}$. and sodium chloride $4.5 \mathrm{~g}$. are also added as electrolytes. The mixture is kept at a temperature of about $55^{\circ} \mathrm{C}$. until, immediately before the injection is carried out, radioiodinated human serum albumin (131I) is introduced in the proportion of 80 microcuries to $500 \mathrm{ml}$. of injection medium and adequately mixed. Before commencing the injection 10-ml. samples of the injection mixture are taken to ascertain the amount of radioactivity per unit volume of the material injected. At the moment of injection the temperature of the mixture is between $45^{\circ}$ and 
$50^{\circ} \mathrm{C}$. Propyliodone aqueous suspension was chosen after a trial of several substances because it is particulate and it blocks the vascular bed at vessels of approximately $30 \mu$ diameter. We have repeatedly confirmed histologically that the propyliodone particles reach the arterioles but do not enter the alveolar capillaries.

A lung that has been carefully removed at autopsy to avoid damage to the pleura is kept as cool as possible in refrigeration until it is warmed for the injection. No lungs have been used that have been taken from bodies more than 36 hours after death. Satisfactory injections have been obtained in lung specimens kept in the deep freeze for a week and then thawed out over an interval of three hours. The results in frozen specimens have been comparable with those carried out immediately in fresh specimens and are in every other way satisfactory. Sometimes it is not possible to remove lungs without tears to the pleura, particularly if one wishes to study the results of earlier thoracic surgical procedures. In these cases it is better to remove the parietal pleura as well and small tears in the pleura can then be sealed with forceps. The preparation of the lung immediately before injection involves bringing it to an even temperature of $50^{\circ}-55^{\circ} \mathrm{C}$. in a water bath, and milking the vessels towards the hilum to remove obvious clots. The injection medium is put in at a pressure of $80 \mathrm{~mm}$. $\mathrm{Hg}$ through a funnel tube and cannula that have been immersed in water at $55^{\circ} \mathrm{C}$. Histological confirmation is obtained that the vessels are fully distended, not showing any evidence of crenation, and that material has not passed through the capillary bed into the venous side. The injected fluid is allowed to enter the lung for as long as it will. Flow usually ceases in less than 3 minutes. It is important to cool the lung as quickly as possible in a cold water bath after the injection has stopped. Inflation of the lung to the limit of lung expansion is then carried out by running "industrial methylated spirit" into the bronchial tree at the lowest pressure commensurate with expansion of the lung. This distension with cold spirit also helps to cool the inner parts of the lung. During these procedures the pressure of the arterial injection is maintained at $80 \mathrm{~mm} . \mathrm{Hg}$ by a sealing clip on the tube near the pulmonary artery. Radiographs are then taken to confirm proper filling of the main branches of the arterial bed and to detect any technical faults in the filling process. It has sometimes been apparent from the radiograph that the injected fluid has burst the vessels and entered air spaces. In such cases the whole lung or the affected part has been discarded. After the radiographs have been taken, a sagital slice $2 \mathrm{~cm}$. thick is taken and pieces approximately 40 cubic centimetres in volume are taken from the periphery of the slice. Samples are taken from comparable areas in each lung, but occasionally from additional areas if there are points of special interest. The volume of each sample is measured by immersing it in a 100-ml. graduated cylinder containing methylated spirit. The radio-activity in each piece is measured by placing it in the centre of a ring of six large geiger counters. The results are expressed in terms of the amount of radioactive medium filling unit volume of the arterial bed.

In the early part of this work we had some difficulty with leakage of radio-activity from the vascular bed into the medium used for distending the air space of the lung. This was particularly so when formol saline was used. This difficulty was overcome by keeping the injection medium between $45^{\circ} \mathrm{C}$. and $50^{\circ} \mathrm{C}$. at the time of actual injection and the lung at only a slightly higher temperature, and by using "industrial methylated spirit" instead of formol saline for distending the air passages; finally confirming that the leak was controlled by taking specimens of spirit from the air passages after distending the lung and assessing the amount of radioactive leak into them. By this method the leakage is so small that it cannot affect the results.

We have accepted the amount of radio-activity per unit volume of lung as an index of the volume of the peripheral small arterial and arteriolar bed in the piece of lung studied.

\section{RESULTS}

The early cases were rejected until we were quite certain that the technique was producing repeatable results. Since then we have been able to study 70 specimens including twelve normal human lungs and three normal lungs from sheep. In man normality of the lung is not always easy to assess but for this purpose we regarded lungs as normal if they appeared normal macro- 
scopically and there was no pathological reason in the lung for right heart strain and no evidence of right heart strain in life. A typical normal radiograph and small artery are shown in Fig. 2. The limits of normality as measured in our cases are shown in Fig. 1. A normal peripheral arterial bed has been found in the lungs of a person over 70 years and such variations as do occur normally do not seem to be governed by age. These limits of normality are strongly supported by the studies carried out in the lungs of sheep in which the figures tend to lie in the higher levels of the normal limits shown. Some of the normal human lungs with figures lying in the lower part of the normal range may well have had slight disorder of lung function of a degree hitherto disregarded as insignificant.

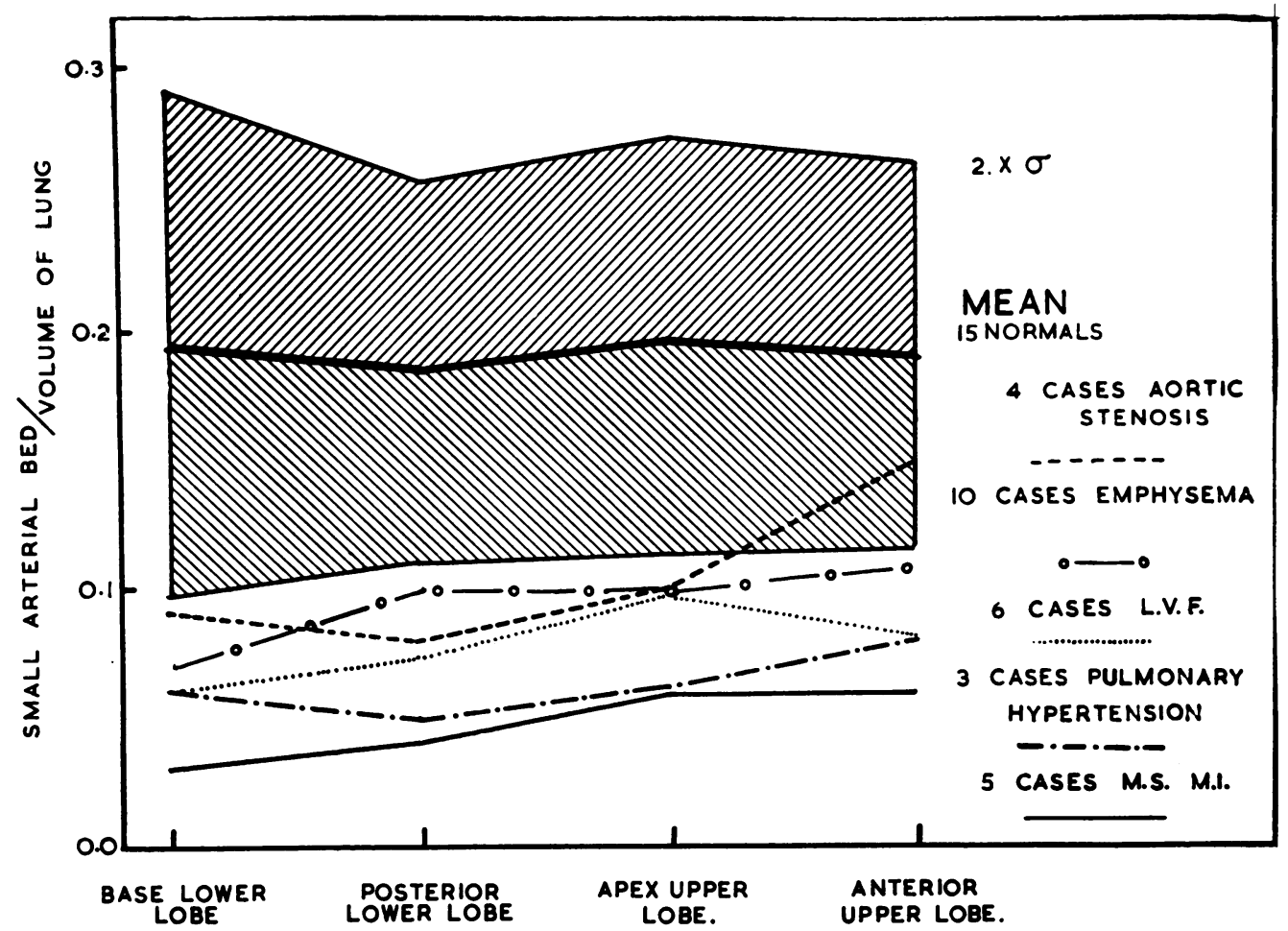

FIG. 1.-Measurement of the volume of selected parts of the peripheral arterial bed expressed in terms of unit volume of distended lung. The mean and the 95 per cent range of the normal lungs (12 human and 3 sheep lungs) are indicated. Reduced small arterial bed is taken as one in which two areas have values below $\mathbf{0} \cdot 10$. The results in mitral stenosis and mitral incompetence (M.S., M.I.), aortic stenosis, left ventricular failure (LVF), pulmonary hypertension, and emphysema are shown.

The intention of this method was to study the reduced peripheral vascular bed in the lung and so we have considered as abnormal those lungs in which at least two areas have shown a reduction below the figure of $0 \cdot 10$ per unit volume. The reason for this choice will be readily seen from the limits of the normal shown in Fig. 1. Within the abnormal group it has been possible to separate the conditions that have given rise to this degree of vascular bed reduction. There is first the well recognized group of mitral stenosis, with or without some degree of mitral incompetence, but with pulmonary hypertension. The cases in this group not only confirm the findings of others but are in accord with the radiographs and the microscopy results. They show that this method gives adequate separation of the abnormal from the lower limits of the normal. The mean of the peripheral arterial bed per unit volume of lung for these mitral valve disease cases is shown in Fig. 1. The scatter about this mean was very small, the highest reading recorded in any part of the five lungs being 0.06. It is clear therefore that in severe mitral valve disease with pulmonary hypertension 


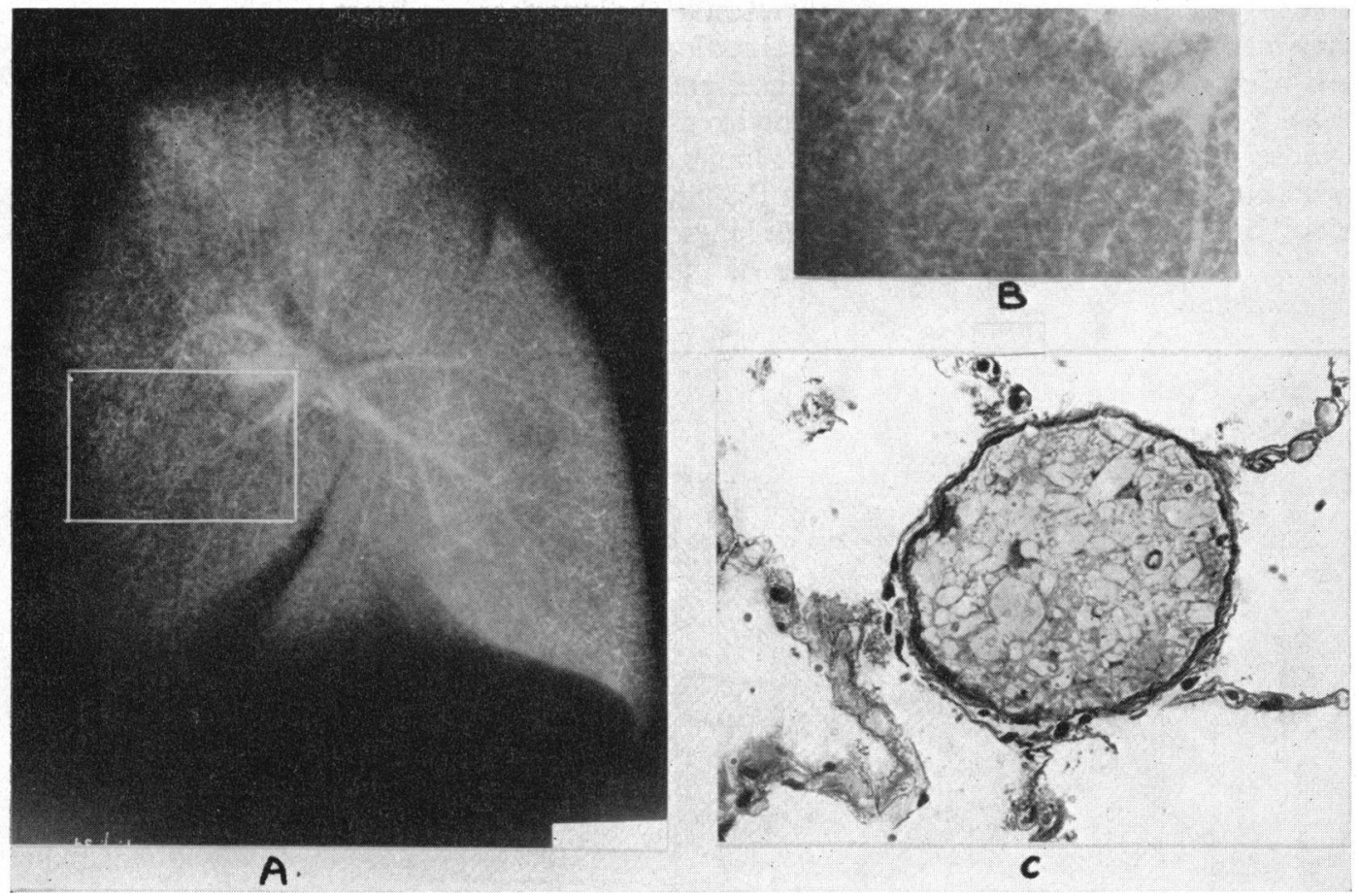

FIG. 2.-No. 51. (A) The injection radiograph of a normal lung from a woman of 40 years who died suddenly from pontine hæmorrhage (readings of $0 \cdot 14,0 \cdot 18,0 \cdot 18,0.20)$. (B) An enlarged view of part of the small arterial bed. (C) A $130 \mu$ vessel shows the normal thickness of the arterial wall relative to the lumen diameter $(\times 405)$. The injection mass in the lumen is well shown.

the separation of the readings from the scatter of the normal is quite distinct. Fig. 3 shows the accepted appearance of the radiograph of the lung and the typical changes in the small arteries.

The first surprise in this work came from the results in the four cases of aortic valve disease, three cases having aortic stenosis and the fourth aortic stenosis and regurgitation with subacute bacterial endocarditis. The figures for these cases indicated a considerable reduction in the peripheral arterial bed, the mean value being indicated in Fig. 1. The reduction is not quite as great as in cases of mitral valve disease but in all four cases parts of the lung have a peripheral arterial bed reduced well below the limits of the normal range, one case in particular having an extreme degree of reduction in the lower lobe. In general in all four cases the reduction is much greater in the lower lobe than elsewhere. Study of the radiographs shows that the pattern is much the same as that seen in mitral stenosis with the bare-tree effect. Histological study of the vessels has shown in all four cases much hyperplasia of the small arteries in the $50 \mu$ to $200 \mu$ range (Fig. 4), similar to that described by Smith et al. (1954). In one of them the thickening of the vessels was very marked and this is consistent with the finding of low figures for the volume of the small arterial bed. There was no evidence at autopsy of mitral valve disease in these cases; cardiac failure was absent in one and in another death was due to subacute bacterial endocarditis rather than to prolonged cardiac failure. Our findings suggest very strongly that there is a reduction in the small arterial bed of the lung in aortic stenosis even before the advent of congestive cardiac failure and this is supported by the histological findings. It has been noted previously that right ventricular hypertrophy occurs unexpectedly in cases of aortic valve disease (Thompson and White, 1936) and we suggest this evidence of pulmonary hypertension as the explanation. Aortic stenosis with enlargement and hypertrophy of the left ventricle, even at a fairly early stage, can produce 


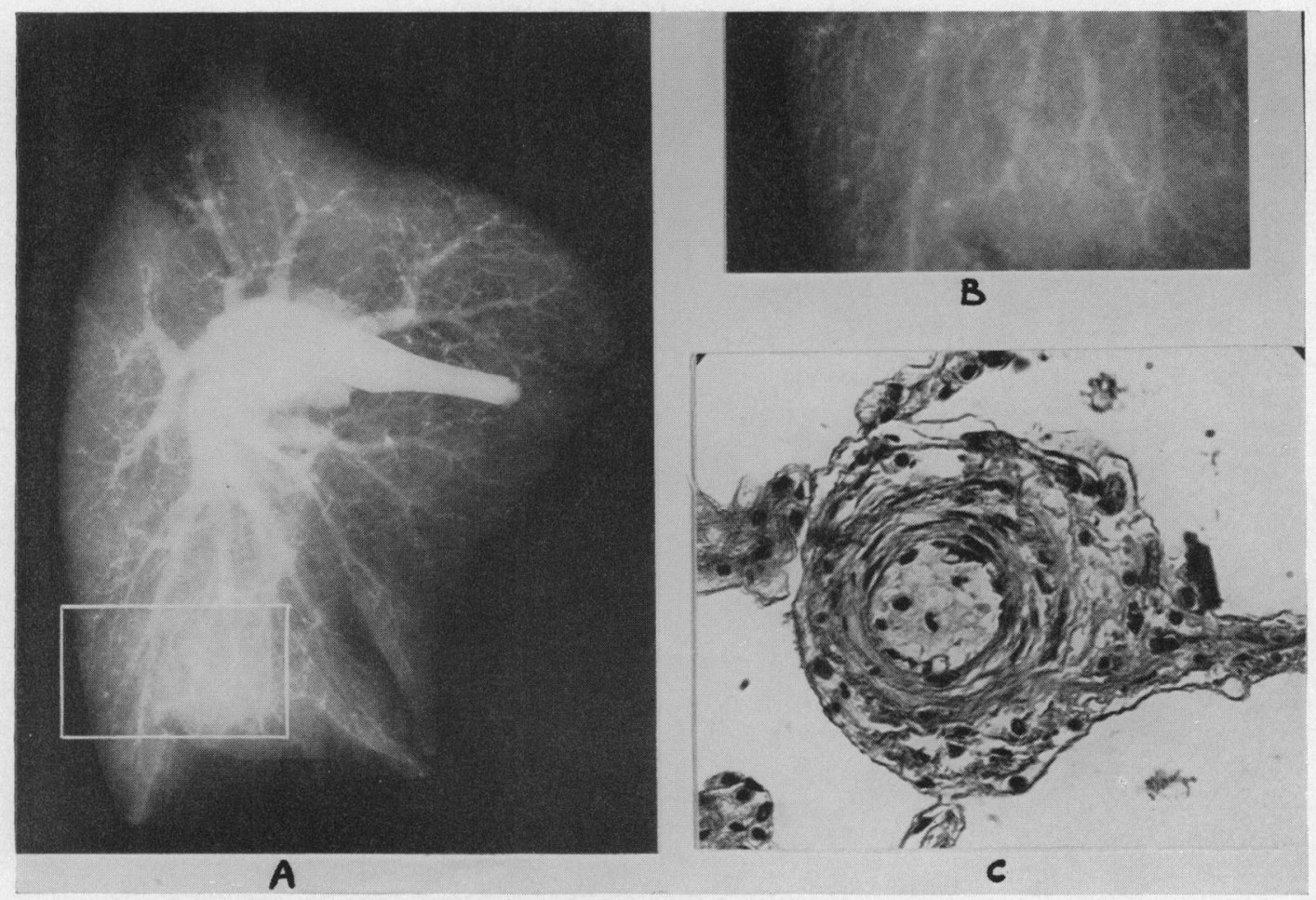

FIG. 3.-No. 27. (A and B) The injection radiograph of a lung from a case with mitral stenosis, present for at least 13 years in a woman of 57 years. The small arterial bed was reduced (readings $0.02,0.05,0.05$, 0.06). The radiograph shows the characteristic bare-tree pattern. (C) A $130 \mu$ vessel illustrates the typical arterial changes $(\times 405)$.

the same form of reduction in the pulmonary vascular bed as mitral stenosis; and if the case proceeds to congestive cardiac failure, the degree of reduction and the change in the vessels will be of the same order as is seen in mitral stenosis. The number of cases we have studied in this group is small but the finding was quite consistent in all of them.

Cases with pulmonary hypertension and right ventricular hypertrophy show great reduction in the peripheral bed. There were three such cases with pulmonary hypertension: a case of patent ductus arteriosus with a pulmonary artery pressure of $105 / 62 \mathrm{~mm}$. $\mathrm{Hg}$; a case with evidence of severe pulmonary hypertension of unknown cause associated with moderate emphysema; and a case of long-standing pulmonary tuberculosis in one lung, treated by thoracoplasty years earlier. In these three cases the reduction of the peripheral arterial bed was great. In the case with a patent ductus the changes in the small arteries of the lung were typical of those described by other authors (Evans and Short, 1958). The second case of pulmonary hypertension had equally severe reduction in the peripheral arterial bed and on histological study changes identical with those seen in mitral stenosis, but there was no heart disease except the severe right ventricular hypertrophy (Fig. 5). In the lungs the changes would normally have been regarded as those of moderate emphysema, and this case will be referred to again in the discussion on emphysema. It is of interest in the case with a thoracoplasty on one side, that the lung on the other side has shown a reduction in peripheral arterial bed consistent with severe pulmonary hypertension. The radiograph shows the same bare-tree appearance and histological study shows evidence of thickening in the small arteries. The severity of the reduction of the pulmonary vascular bed in these three cases of pulmonary hypertension is well shown by this method, and a clear explanation of the severe right ventricular hypertrophy is provided, though initially it was unexplained in the second and third cases. 


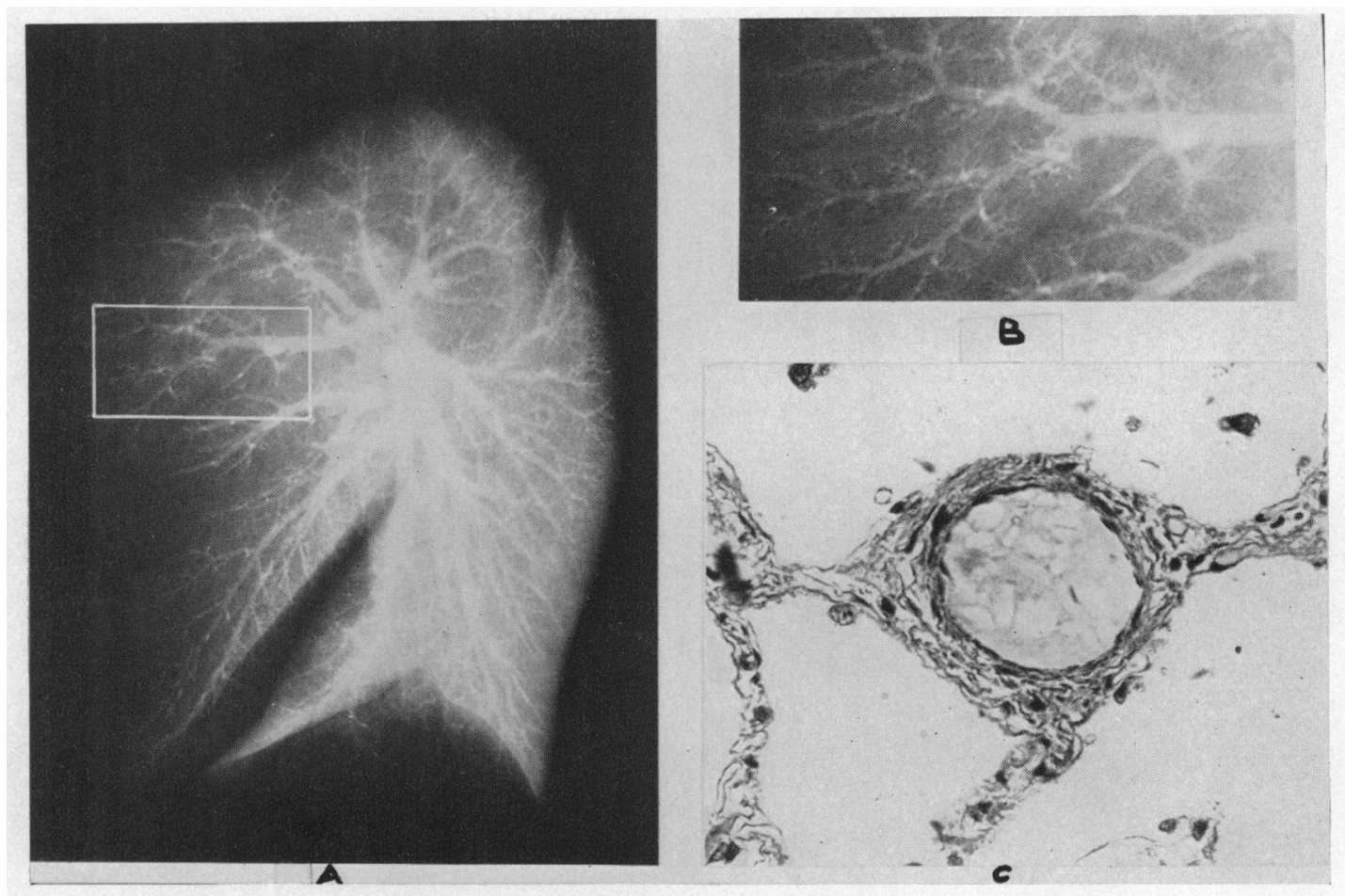

FIG. 4.-No. 36. (A) The injection radiograph of the lung in a case of aortic stenosis and regurgitation (dominant stenosis) in a man 35 years of age who died of subacute bacterial endocarditis (readings 0.03 , $0 \cdot 05,0 \cdot 10,0.09$ ). (B) The radiograph shows a bare-tree pattern. (C) The $110 \mu$ arteriole illustrates mural thickening which was present in the areas of reduced arterial bed $(\times 405)$.

The next group, which is different from the others, is that of left ventricular failure occurring in cases with systemic hypertension or with coronary heart disease. There are six such cases in which there was unequivocal hypertrophy of the left ventricle and evidence before death of left ventricular failure. The mean for these cases is indicated in Fig. 1. There is a fair degree of scatter in the figures as would be expected because they vary in severity, the figures in the more severe cases for the entire lung being 0.07 or below and of the same order as severe mitral stenosis. This finding is surprising but, as in the case of aortic valve disease, it explains the presence of right ventricular hypertrophy in some of these cases. Reviewing the history it would seem that if the left ventricular failure had been present for periods as short as a few weeks, then there was a likelihood that the small arterial bed in the lung would be reduced. The histological search for supporting evidence has shown in the case with the most severe reduction that there were changes in the $50 \mu$ to $100 \mu$ diameter vessels (Fig. 6). There is no doubt that where left ventricular hypertrophy and dilatation has existed for a period of time, a reduction has taken place in the small arterial bed of the lung, and even if left ventricular failure has been present for only a few weeks measurable changes can be found by this method of studying the peripheral arterial bed.

The last and perhaps the most intriguing group to be considered is that of emphysema. The surprising finding in the study of the 10 cases of emphysema has been that in those regarded macroscopically as having slight or moderate emphysema, this method has shown considerable reduction in the peripheral arterial bed in affected parts of the lung (Fig. 7 and 8). There are 5 cases of the 10 in which the emphysema, regarded macroscopically as moderate, seems to be the only possible explanation for finding quite severe restriction of the small arterial bed with some evidence of pulmonary hypertension. In the cases with slight or moderate emphysema there are 


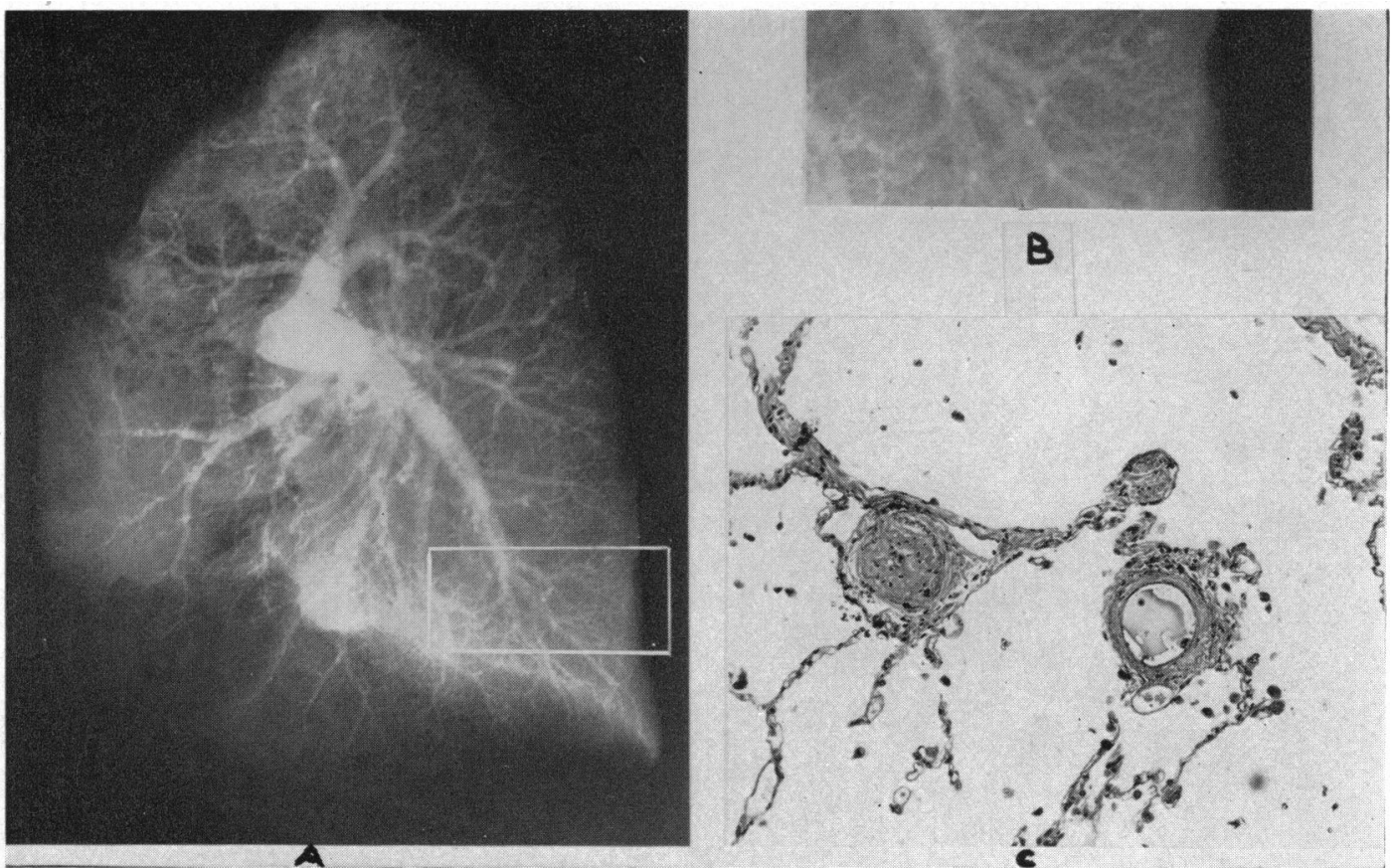

FIG. 5.-No. 19. (A) The injection radiograph of the lung from a case of pulmonary hypertension with gross right ventricular hypertrophy without established cause in a woman of 55 years. There was moderate emphysema present but hardly sufficient to explain the vascular changes (readings $0.04,0.03,0.04,0.04$ ). (B) A bare-tree pattern is shown in the enlarged portion. (C) Both arterioles have a maximum diameter of $140 \mu(\times 130)$, one being obliterated and the wall of the other thickened. These changes were general throughout.

areas of the lung that give low normal values and other areas, the emphysematous areas, with considerable reduction below normal in values. We have considered a possible source of error in that emphysematous bullæ are liable to collapse and therefore the volume estimation is not accurate. In the cases studied the blocks have been taken from areas in which collapse has not taken place on cutting. Lungs with large bullæ are now being distended with a solution of ionagar to keep the true volume of the pieces when cut. Histological search in the areas showing considerable reduction of the pulmonary arterial bed has shown that the small vessels have become thickened and narrowed, and can eventually be traced to complete occlusion and transformation into fibrous cords. In an ordinary search of the lung field the missing vessels would not be recognized and their number could not be assessed except by this method. It is also noticeable that only in emphysema does the injection medium repeatedly break the vessels and enter the air space. It has been possible by this measurement of the small arterial bed to identify areas of moderately emphysematous lung in which the reduction of the arterial bed has been considerably more than suspected. This has provided a stimulus to further detailed study of the subject.

A number of other conditions have been studied singly. The only lung with pure asthma examined revealed a perfectly normal pulmonary arterial bed, although the asthma had been present for thirty years. This agrees with other findings of ours which suggest that pure asthma does not alter the arterial bed permanently, but the addition of bronchitis and emphysema will change it. A case of repeated small embolization of the lung gave very low values for the peripheral arterial bed as would be expected. A case of epilepsy with death from drowning gave very low figures for the arterial bed but there was right ventricular hypertrophy so there must have been, pulmonary hypertension of some duration as yet unexplained. One lady who lived to the age of 72 


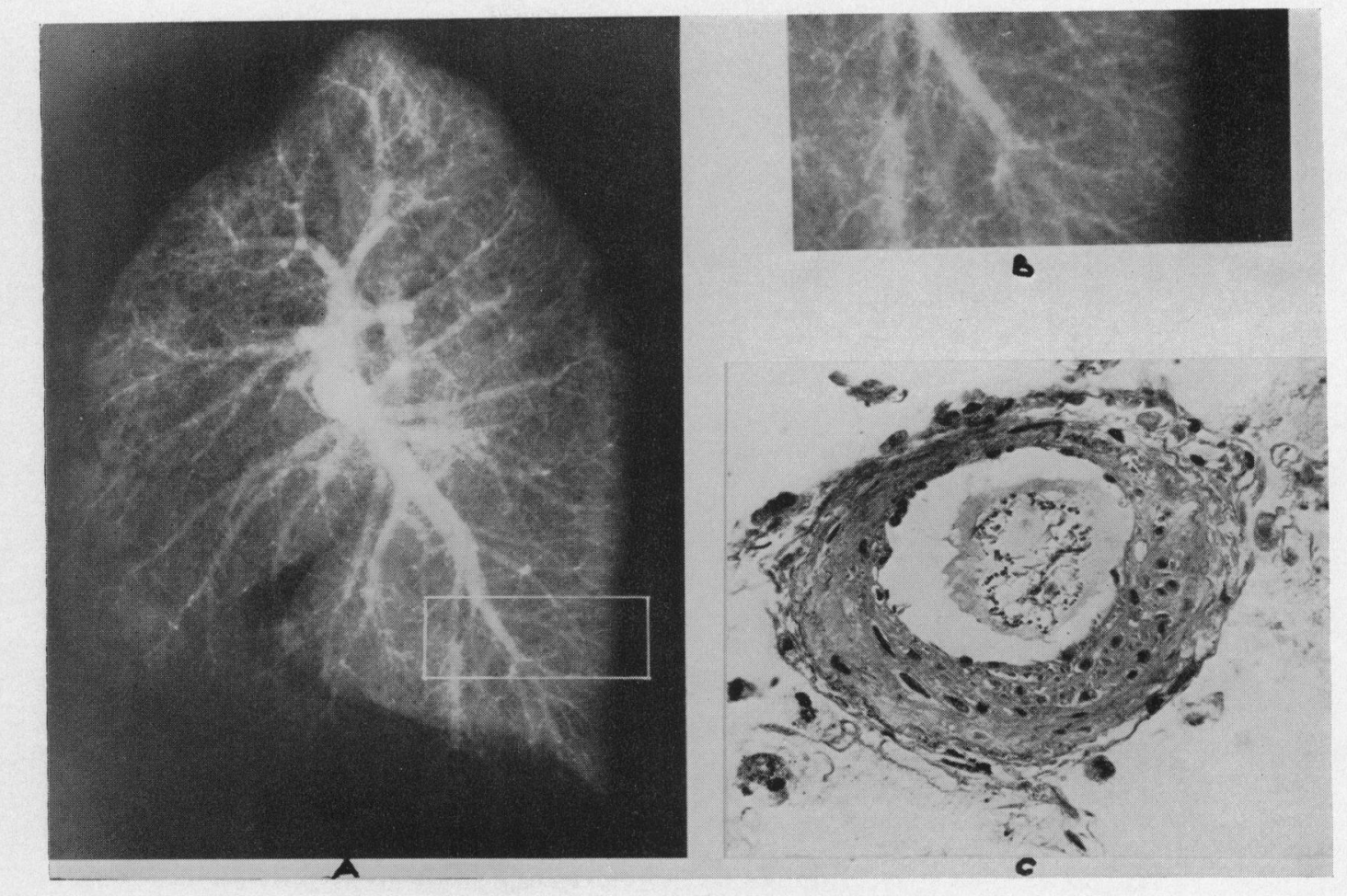

FIG. 6.-No. 46. (A) The lung from a man of 71 with exertional dyspnœa for four years and terminal left ventricular failure after cardiac infarction. The heart weighed $540 \mathrm{~g}$. with severe left ventricular hypertrophy. The readings $0.07,0.07,0.10,0.09$, indicate considerable reduction in the small arterial bed. (B) There is considerable pruning of the vascular pattern. (C) There is obvious gross thickening in the wall of many small vessels; the one shown is of $190 \mu$ diameter $(\times 405)$.

years with mitral stenosis proved to have a peripheral arterial bed in the lower limits of the normal range, perhaps explaining her long survival.

\section{Discussion}

Lewis et al. (1953) have shown that cardiovascular conditions affecting primarily the left ventricle can give rise to an increase in pulmonary capillary pressure on exercise or in more severe cases at rest. They have shown that this results in an increase of pulmonary arterial pressure and right ventricular work. Smith et al. (1954) have described changes in the muscular arteries and arterioles in these conditions. Goodwin (1958) discussing the nature of pulmonary hypertension has emphasized the part played by increase in left atrial and pulmonary venous pressures.

The present work has given a quantitative record of the reduction of the small arterial bed in cases of aortic valve disease, hypertension, and coronary artery disease, in some of which the history was simply that of dyspnœa on effort. In the case of subacute bacterial endocarditis there was a state of moderate heart failure for a period of three weeks only and in a case of cardiac infarction there was a short history of attacks of left ventricular failure. A large reduction of the small arterial bed has been demonstrated in these cases even before there has been time for gross histological change to take place. This reduction in the vascular bed may be the result of the elevation of the post-capillary venous pressure.

The injection method showed that the small arterial bed was reduced in emphysema. A reduction was demonstrable even when the amount of emphysema judged by naked eye was slight. The disappearance of alveolar walls and of capillaries is well recognized in emphysema, and thickening 


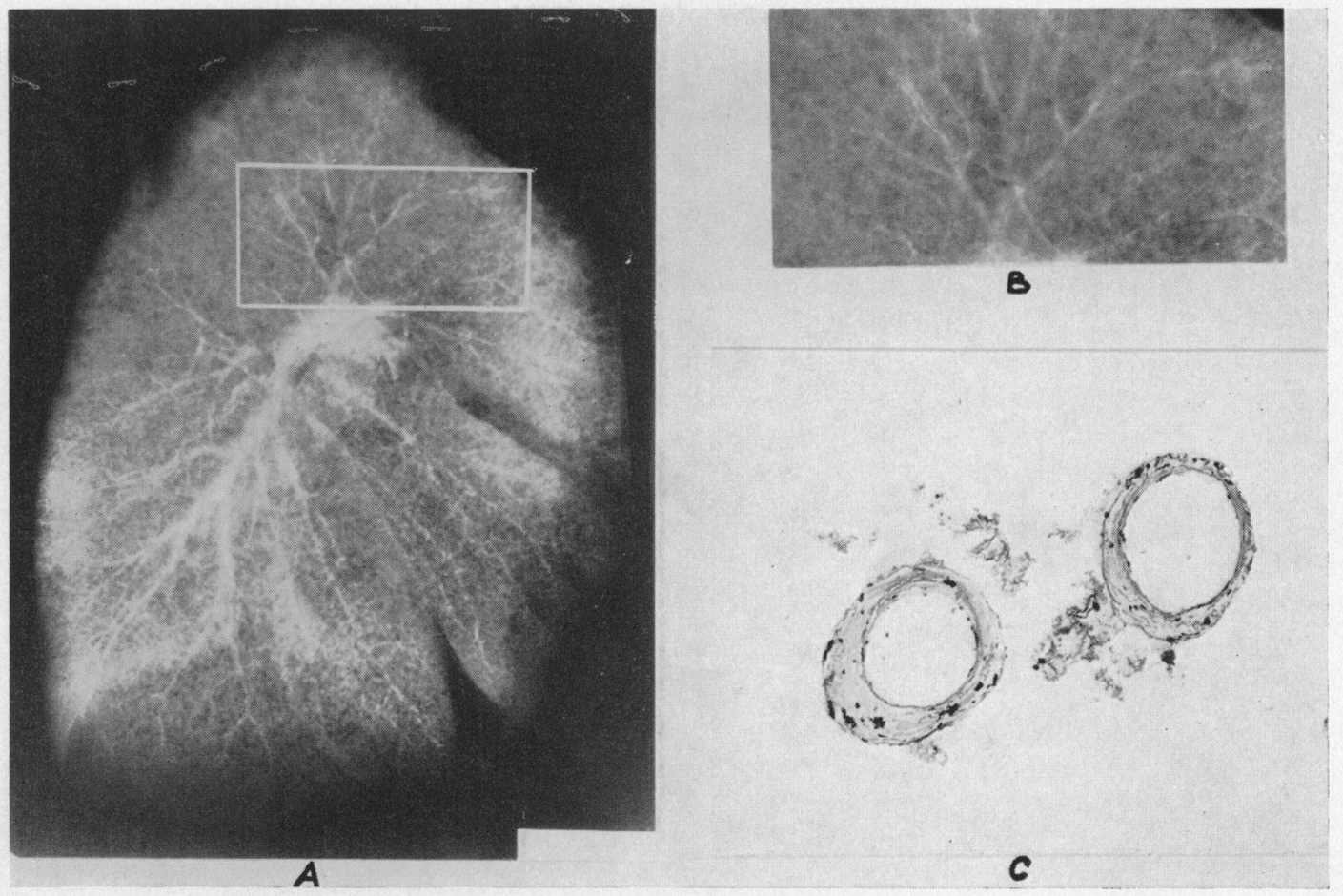

FIG. 7.-No. 26. Severely emphysematous lungs in a man of 63 years. (A) The injection radiograph shows disappearance of smaller vessels and in several areas there has been leakage into the air spaces. The readings of $0.04,0 \cdot 17,0 \cdot 10,0 \cdot 12$ show the variability of the reduction. (B) The enlargement shows more clearly the features seen in the emphysematous lung. (C) Both vessels shown are about $180 \mu$ in maximum diameter. They are ill-supported arterioles with thickening of their walls $(\times 160)$. (See also Fig. 8.)

of small vessels has been described. This method has the advantage of measuring the amount of vascular loss as well as the reduction in volume of existing vessels. The manner in which the reduction of the small arterial bed takes place differs from that in the left heart conditions. There is a narrowing of small arteries with some thickening of their walls in the areas in which capillaries are being damaged, thus reducing blood flow to the damaged areas. These small unsupported vessels may break in time but often persist as occluded fibrous cords. Our measurements suggest that considerable reduction of arterial bed occurs even in moderate emphysema. No evidence of shunts over $30 \mu$ in size has been found in the absence of bronchiectasis. A suggestion is made that damage to the capillary bed acts in the same way as a raised capillary pressure causing "shut-down" in the small arterial bed to protect the threatened area.

It should be possible to confirm the results in left ventricular failure and in emphysema by reproducing these conditions experimentally. This is being attempted and will be described separately but the work to date supports the finding that "shut-down" of the small arterial bed can be demonstrated only a few hours after the appearance of left ventricular failure. Another source of confirmation of this work is the wedge pulmonary arteriography described by Bell et al. (1959).

\section{SUMmary}

In the study of the pulmonary vascular bed by injection methods, assessment hitherto has been visual. A method incorporating a radioactive substance with the radio-opaque medium has enabled measurements of the small arterial bed $(2 \mathrm{~mm}$. to $30 \mu$ vessels) to be made. The measurement is 


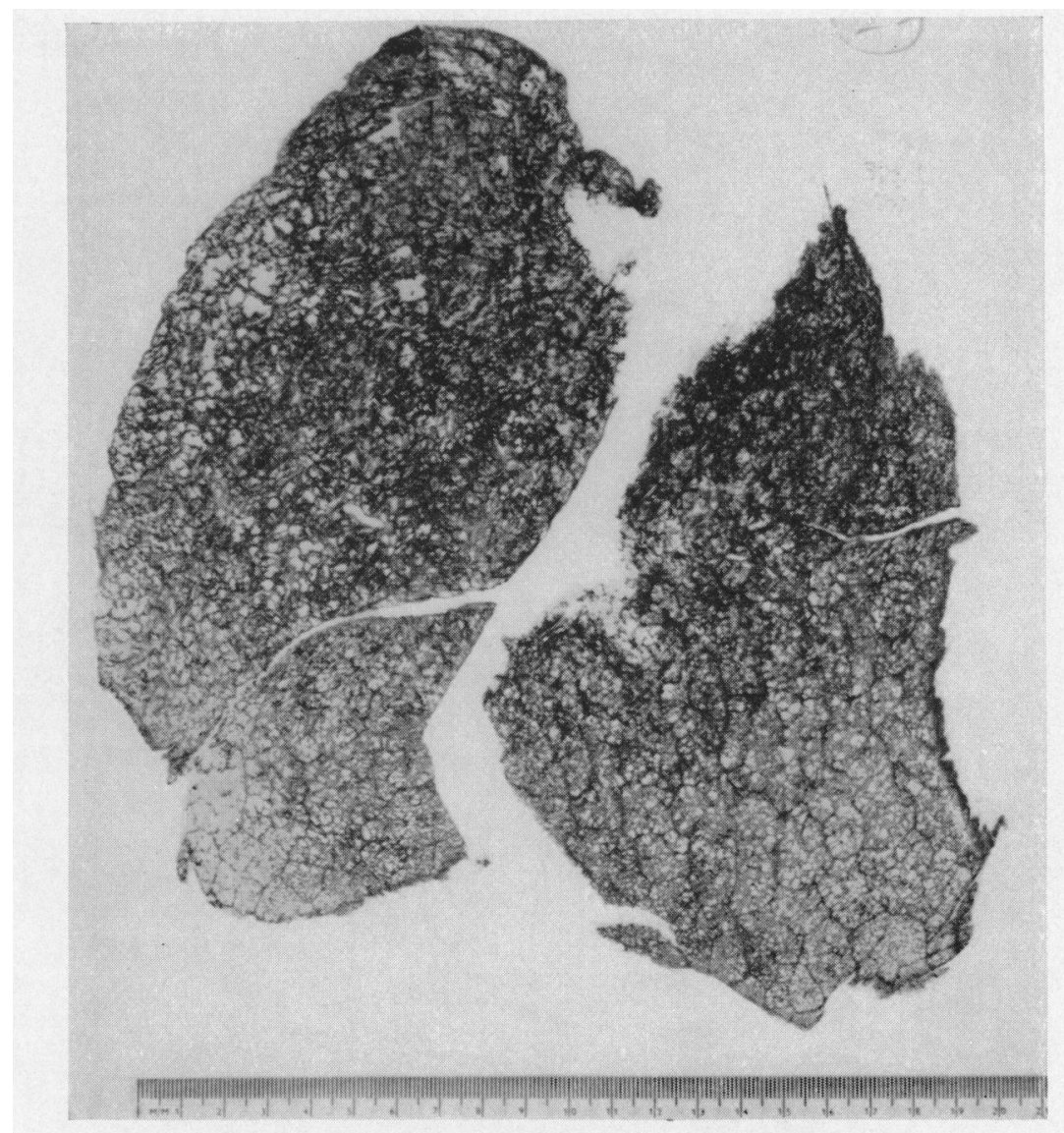

FIG. 8.-The lung section (Gough-Wentworth technique), showing the extent of emphysema in the lung shown in Fig. 7.

expressed in terms of volume of small arteries per unit volume of lung and the mean and the range for normal lungs has been identified. Lungs with a reduced vascular bed have been grouped according to the causes, such as mitral valve disease, aortic valve disease, pulmonary hypertension, left ventricular failure, and emphysema. The method has shown much reduction of the small arterial bed of the lung in cases of aortic valve disease and of systemic hypertension and cardiac infarction with left ventricular hypertrophy. This reduction takes place at a very early stage of left ventricular failure or possibly even before this occurs. There is already some experimental support for this view.

An analysis of 10 cases of emphysema is presented and the results indicate that the reduction in the small arterial bed is much more than the macroscopic appearance of the emphysema would suggest. Smaller vessels have disappeared while many of those remaining have thickened walls with narrowing of the lumen. This method measures the total reducing effect of these changes.

The findings in the differing groups of cardiac and respiratory disease are in accord with the view that elevation of capillary pressure or damage to the capillary bed acts as an immediate stimulus to restriction of the associated small arterial bed to protect the capillaries. Such reduction may be identified by measurement before it has produced changes that can be confidently observed by the microscope.

We wish to thank the pathologists of the United Cardiff Hospitals, St. David's and Sully Hospitals for providing us with interesting material. Our thanks are also tendered to Dr. Glyn Evans of the Radiotherapy Hospital; Mr. 
Williams, pharmacist; Mr. Leighton Williams, medical photographer of Llandough Hospital; and Mr. Travers, medical photographer to the Institute of Pathology, Welsh National School of Medicine. The animal experimental work is being done with the help of the Animal Experimental Laboratory at Sully Hospital.

\section{REFERENCES}

Bell, A. L. L., Schimomura, S., Taylor, J. A., and Fitzpatrick, H. F. (1959). Progress in Cardiovascular Diseases, $2,64$.

Borst, H. G., Berglund, E., and McGregor, M. (1957). J. clin. Invest., 36, 669.

Brenner, O. (1935). Arch. intern. Med., 56, 211.

Davies, L. J., Goodwin, J. F., Steiner, R. E., and Van Leuven, B. D. (1953). Brit. Heart J., $15,393$.

Evans, W., and Short, D. S. (1958). Brit. Heart J., 20, 529.

Goodwin, J. F., Steiner, R. E., and Lowe, K. G. (1952). J. Fac. Radiol., 4, 21. (1958). Brit. J. Radiol., 31, 174.

Lewis, B. M., Houssay, H. E. J., Haynes, F. W., and Dexter, L. (1953). Circulat. Res., 1, 312.

McKeown, F. (1952). Brit. Heart J., 14, 25.

Motley, H. L., Cournand, A., Werko, L., Himmelstein, A., and Dresdale, D. (1947). Amer. J. Physiol., $150,315$.

Parker, R. L. (1940). Ann. intern. Med., 14, 795.

Short, D. S. (1957). Lancet, 2, 12.

Smith, A. C., Burchell, H. B., and Edwards, J. E. (1954). Circulation, 10, 801.

Thomas, A. J., James, W. R. L., and Owen, G. M. (1959). Lancet, 1, 1024.

- - (1958). Brit. Heart J., 20, 403.

Thompson, W. P., and White, P. D. (1936). Amer. Heart J., 12, 641. 